\title{
Influence of Basic Environment of Geopolymer Composites on Degradation Rates of E and AR Type Glass Fibers
}

\author{
Martina Ryvolová, Lucie Svobodová, Totka Bakalova, Lukáš Voleský \\ Faculty of Mechanical Engineering, Technical University of Liberec, Studentská 1402/2, 461017 Liberec, Czech \\ Republic.E-mail: martina.ryvolova@tul.cz; lucie.svobodova@tul.cz; totka.bakalova@tul.cz, lukas.volesky@tul.cz
}

In our research, the corrosion mechanism on the surface of anti-corrosion glass fibers of type AR and glass fibers of type $\mathbf{E}$ was studied. The monitoring was performed under the influence of an alkaline environment. The glass fibers were placed in a solution of calcium hydroxide $\mathrm{Ca}(\mathrm{OH})_{2}$ for a specified period of 2, 4, 6, 12, 24, 48 weeks. The change in mechanical properties was monitored; basen on test ĆSN ISO 695 was evaluate the durability and resistance of E-GF and AR-GF to corrosion. The degradation resistance of the fibers was evaluated using a tensile strength test (ASTM D2343-17). The use of the investigated fibers (as reinforcement in a geopolymer composite) led to a $250 \%$ increase in flexural strength for AR-GF; for the E-GF sample, the flexural strength increased by $150 \%$. The deflection of the reinforcing geopolymer composite increased by $180 \%$ compared to the pure geopolymer mixture (for both types AR and E). The surface morphology of the fibers was characterized by scanning electron microscopy (SEM). Quantitative evaluation of the corrosion mechanism was performed through modern procedure based on image analysis (Matlab software).

Keywords: Anticorrosion glass fibres, geopolymer composite, corrosion process, strength, image analysis

\section{Introduction}

From a chemical point of view, glass fibres (GFs) belong to the group of inorganic fibres, which include metal fibres (e.g. silver, copper, steel) and their alloys, non-metals (e.g. carbon, boron fibres) and mineral fibres (e.g. basalt). Glass fibrils are the product of a melting process. Melt (its input raw materials are glass sands - $\mathrm{SiO}_{2}$, rocks containing $\mathrm{Al}_{2} \mathrm{O}_{3}$, ground limestone - $\mathrm{CaO}$, soda or potash $\mathrm{Na}_{2} \mathrm{O}$ and $\mathrm{K}_{2} \mathrm{O}$ and other auxiliary substances with specific effects) creates by heating suitable raw materials to a temperature of about $1500{ }^{\circ} \mathrm{C}$, following by its controlled cooling. The process of drawing from a platinum furnace forms continuous fibril. The next step is the lubrication of fibrils. Sizing protects them and allows their manipulation and processing. Fibrils are grouped into so-called rovings (a longitudinal textile with a defined number of fibrils) and wound into cocoons [1]. The following step is preparation of different types of composite reinforcements. Short glass fibrils are made by centrifuging or blowing the melt. According to the quantity of oxides, we dis-tinguish between different types of glass fibres, which differ in their properties and technical use. Both variants of glass fibres - continuous and short, have their technical application. Short fibres are used mainly in construction as a filler for concrete mixtures, which prevents the formation and propagation of cracks during shrinkage of concrete. For these applications, it is necessary for the glass fibres to resist strongly alkaline environments.
This can be achieved either by a special composition of the glass stem (using glass fibres of the AR type (AR-GFs) anti-corrosion glass fibres [2]), or by a suitable surface treatment of fibrils. Continuous $\mathrm{E}$ glass fibres (E-GFs) are used directly for the production of composites - fibre-reinforced plastics and for the production of composite reinforcements (for example woven, knitted or braiding fabrics) [3].

The term degradation generally means deterioration or decline existing material properties. In the case of glass fibres, degradation can be quantified as weight loss and a consequent reduction in fibre strength due to an acidic [4] or alkaline environment [5]. The alkaline environment erodes the bonds between the silicon atoms. The fibres dissolve under prolonged exposure to temperature and high $\mathrm{pH}$ [2]. Laboratory tests to simulate for accelerated aging are used for determination of degree of degradation the fibres at designated conditions. Ongoing corrosion processes lead to a reduction in the mechanical properties of the fibers (in the case of their use as reinforcement in the composite) and at the same time the mechanical properties of the geopolymer composite [6]. Generally, many studies confirmed this fact $[7,8,9]$. Morphological changes on the surface of the fibers indicate ongoing degradation processes. Typical changes are the formation of small aggregates, holes, cracks or the formation of a continuous coating of the so-called corrosion shell [10].

Effect of fibre surface texture on the mechanical properties of glass fibre reinforced epoxy composite 
was described in [11], where the volume of damaged material at each stage of penetration was quantified; the sizing formulations varied the degree of chemical bonding and modified the fibre surface texture in the sizing. An automatic classification of composite materials surface was studied in [12]; where the wavelet texture analysis served to the automatically classifying of carbon fibre reinforced plastic samples. Generally, in the science literature, only the diameter of fibres was determined and seldom the fiber texture was monitored but not analysed. The formation of a brittle, shell-like layer (corrosion layer) was observed on the filament surface after washing in $5 \% \mathrm{NaOH}$ solution [6]. The corrosion shell peeled off created a smooth "fresh" surface [5]. The morphology of the fibre surface was significantly affected by solution concentration (the higher temperature and the higher solution concentration accelerate the formation of the corrosion shell) [8, 13-15].

The aim of the research is to investigate and compare the rate of degradation processes on the surface of monitored fibers (AR-GFs and E-GFs). Moreover, the comparison between mechanical properties [16] and surface observation (based on SEM microscopy and image analysis) was carried out; these results show inverse linear relationship that was not described in previous papers.

In addition, the work is focused on verifying the suitability of using image analysis as an equivalent method of evaluating ongoing degradation processes. A novelty in the research is the use of the image analysis method for quantification of results, i.e. the method offers measurable values, which are then statistically evaluated and processed.

\section{Experimental methods}

\subsection{Materials}

Tab 1 Parameters of geopolymer composite samples

\begin{tabular}{|l|l|l|l|}
\hline & Geopolymer mixture* & Fibres / weight ratio & Weight (average of 10 samples) $[\mathrm{g}]$ \\
\hline I. & $1: 0.9: 1$ & without fibres & 174.4 \\
\hline II. & $1: 0.9: 1$ & AR-GF $/ 2 \%$ & 184.6 \\
\hline III. & $1: 0.9: 1$ & E-GF $/ 2 \%$ & 207.8 \\
\hline
\end{tabular}

* Geopolymer mixture describe weight ratio of geopolymer ingredients - part A: part B: filler.

\subsection{Methods for determination the degree of degrada- tion of GFs}

For determination the durability and resistance of E-GFs and AR-GFs against corrosion was carried out test according to Standard ČSN ISO 695 - Alkali resistance [23]. Glass fibres was deposited in alkaline liquid $\mathrm{Ca}(\mathrm{OH})_{2}$ to a defined time $(2,4,6,12,24,48$ weeks). The resistance of the fibres to degradation was evaluated using tensile strength test (Testometric device, according to Standard ASTM D2343-17 [24]. Clamping length between mechanical jaws was set at
AR-Glass roving is product from producer Owens Corning (Belgium). Nominal linear weight of roving Cem-FIL 5325 is 2400 tex, specific gravity $2.68 \mathrm{~g} / \mathrm{cm}^{3}$, modulus of elasticity $72 \mathrm{GPa}$, tensile strength 1000 $1700 \mathrm{MPa}$.

E-Glass roving (producer Johns Manville, Slovakia) is signated PR440 2400907, nominal linear weight 2400 tex, specific gravity $2.68 \mathrm{~g} / \mathrm{cm}^{3}$, modulus of elasticity $70 \mathrm{GPa}$, tensile strength $1200-1700 \mathrm{MPa}$

BAUCIS LK (České lupkové závody, a.s.; Czech Republic) was used also geopolymer composite mixture for samples preparation. Baucis is inorganic, alluminosilicate binder based on metakaoline (part A) usually activate alkaline activator (part B). Suitable filler are sands with different diameter of particles. Specific gravity $1.8-1.9 \mathrm{~g} / \mathrm{cm}^{3}$, workability $60 \mathrm{~min}$, compressive strength $60 \mathrm{MPa}$, temperature resistance is in range from $-30{ }^{\circ} \mathrm{C}$ to $+120^{\circ} \mathrm{C}$.

For determination degree of degradation of glass fibres in alkaline environment was used $1 \mathrm{~mol}$ liquids $\mathrm{Ca}(\mathrm{OH})_{2}$.

\subsection{Samples preparation}

Preparation of glass rovings samples for tensile strength test was carried out according to the Standard ISO 11566(1996) [18]. Using of paper frames allowed better manipulation with samples and damage of rovings elimination. All tested groups contained 10 samples for possibility of statistic evaluation of the results.

For testing mechanical properties of geopolymer composite reinforced with glass fibres was selected three-point bending test (ISO 14125 [19], ČSN EN 14617- 2 [20]) and impact (Charpy) test (ČSN EN 14617-9 [21], EN 10045-1 [22]). For both methods were prepared samples with prism shape about size

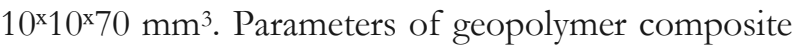
samples present Table 1.
$100 \mathrm{~mm}$, test speed $10 \mathrm{~mm} / \mathrm{min}$. The equipment Testometric used made it possible to evaluate the highest values of strength and Young's modulus of elasticity. At the same time, a graphical record of the course of each test was created.

Samples of composite material were prepared without reinforcement and with $2 \%$ weight ratio EGFs and AR-GFs reinforcement (length of fibres $12 \mathrm{~mm}$ ). The three-point bending tests were conducted on ten specimens with dimension $10^{\times 1} \times 100 \mathrm{~mm}^{3}$ for each materials combination, span 
of supports was $80 \mathrm{~mm}$. Impact resistance was measured on five samples with same composition using Charpy's tester with a $4 \mathrm{~kg}$ hammer.

For evaluation of measured data was used following equations:

- three-point bending test:

$$
\sigma=\frac{3 F l}{2 h b^{2}}
$$

where $\sigma[\mathrm{MPa}]$ - Flexural Strength, F $[\mathrm{N}]$ - Maximal Load, $l[\mathrm{~mm}]$ - Support Span, $h[\mathrm{~mm}]$ - Thickness of Sample, $b[\mathrm{~mm}]$ - Width of Sample.

- Charpy test:

$$
\sigma_{C h}=\frac{E}{A}
$$

where $\sigma_{C b}[\mathrm{MPa}]$ - Impact Strength, E J] - Impact Energy Necessary for Break of Sample, $A\left[\mathrm{~mm}^{2}\right]-$ Cross-section of Sample.

As a degradation layer (for the needs of image analysis method evaluation) was defined the space between the fibre surface and the matrix (in this case a geopolymer matrix). The main parameters of degradation in an alkaline environment that affect the composition of the layer are time, temperature and concentration of the solution. The surface of fibres (without

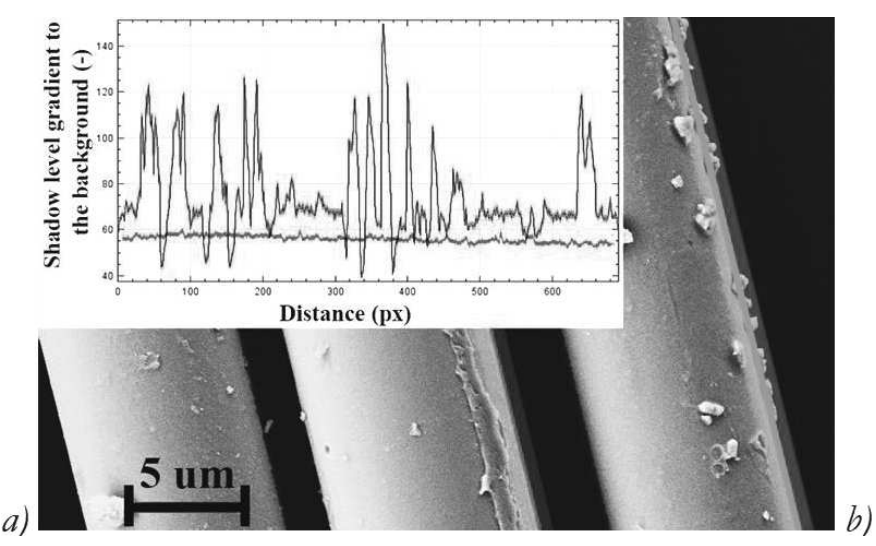

Fig. 1 Image documentation of the calculation process: (a) thickness of the degradation layer, (b) surface texture. Red line/ area shows the fibre with smooth surface; on the other hand, the green line/ areas show more pronounced texture with clusters formed on the fibres surface

The fibre surface morphology was characterized using scanning electron microscopy (SEM). The glass fibres type of $\mathrm{E}$ with sizing appear to have a very smooth surface, while the sizing possesses a more prominent texture with clusters formed on the anticorrosive fibres (AR-GFs) surface. Matlab (The MathWorks) software was used for image analysis [17]. Following steps were conducted. (a) The functions 'adapthisteq' and 'imadjust' was applied with the aim to correct the failures in image, then the images were converted to a gray-level form. (b) The morphological operations 'bwareaopen' followed with 'imclose' reduce another defect (e.g. image capture errors, dust

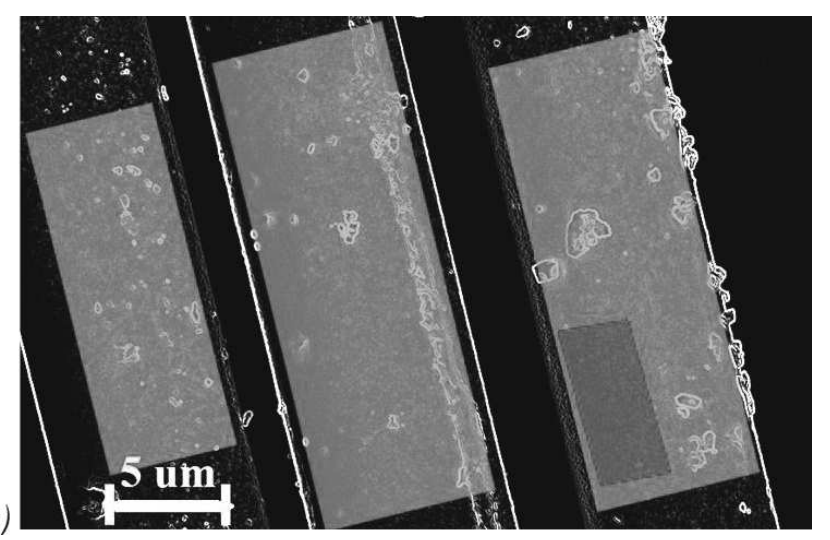

the influence of the environment) is usually treated by sizing. This layer protects the surface before damage, allows the processability/workability of the fibre and helps to create a synergistic bond between the fibre (dispersion part of the composite) and the matrix (continuous part of the composite); helps to create an interfacial interface with all its components (interphase and interface). Over time, the sizing gradually dissolves. After dissolving the sizing, the alkaline environment begins to act directly on the glass fibre structure. The hydroxyl ions attack silica chains and insoluble hydroxides create. Mineral incrustations begin to form on the surface of the fibre and contains this element. Incrustations are at first sporadic, later they begin to coalesce into a continuous layer. The result is a corrosion coating / corrosion shell. It is very important to distin-guish whether the degradation layer contains sizing and its residues and already contains mineral incrustations. SEM analysis is able to show the shape and size of objects on the surface of the fibres, not their chemical composition. To detect the aging phase, it is necessary to design and test a suitable method/s that will complement the image analysis and help determine approximately what stage of aging the fibre is in. 
line). Degradation layer thickness is calculated as an average values of shadow gradient (graph on Figure 1a). (e) Surface texture is calculated on fibre surface as show on Figure $1 \mathrm{~b}$ (green and red areas). The edges detection process (calculates the gradient using the derivative of a Gaussian filter) find the edges (areas) with the local maxima of the gradient, Figure 1b, only edges are displayed as white on black background). All pixel values inside of the green area (Figure 1b) are calculated (averaged and standard deviation) for each fibre in each image. (f) Defined parameters were subjected to statistical analysis. Selected parameters (Figure 5 and Figure 6) are expressed in percentage in relation to the reference sample (sample in zero-time, that is equal to $100 \%$ ), occasionally no measurement error is showed because of high amount of outlier's measurement (due to the great nonuniformity in the images due to the fiber sizing or corrosion shell formation). Box plots show averages values, variance values and also the outlier's data.

\section{Results and discussion}

\subsection{Evaluation of mechanical properties}

The topic of the article is to compare the mechanical properties and define the differences in the degradation process for anticorrosive glass fibres type AR and for glass fibres type $\mathrm{E}$ after influence of the alkaline environment in the geopolymer composite. The ongoing reactions between glass fibres and alkaline environment lead to the dissolution and slowly decomposition of the fibres and consequently to the loss of the mechanical properties of the composite. Corrosion of glass fibres is characterized by weight loss, change of fibre diameter, reduction of mechanical properties and formation of mineral incrustations on the surface of the fibre, which gradually leads to the formation of a continuous corrosion coating so called corrosion shell. Scanning electron microscopy was used to monitor the morphology of the fiber surface, and image analysis methods were used to evaluate morphological changes. Tests of mechanical properties (fibers - tensile test, composite materials - threepoint bending and Charpy test) were used to confirm ongoing degradation processes due to the long-term influence of the alkaline environment on both types of glass fibers.

The measured average values the strenght for both types of glass rovings (AG-GFs and E-GFs) before the action of the basic solution are similar. Strength of AR-GFs was measured $811.4 \pm 12.3 \mathrm{~N}$, strength of $\mathrm{E}$ GFs $825.2 \pm 33.4 \mathrm{~N}$. The measured values of tensile strength of E-GF rovings, after long-term exposure to alkaline medium ( $1 \mathrm{~mol}$ of liquid $\left.\mathrm{Ca}(\mathrm{OH})_{2}\right)$, show a decrease in mechanical strength. Graph in Fig. 2 shows this trend.

Table 2 presents the results of three-point bending test and compares flexural strength geopolymer composite samples without glass reinforcement (pure) and with different type of glass reinforcement. The use of fibres as reinforcement in the composite led to an increase in flexural strength of $250 \%$ for samples with AR-GFs, for E-GFs increased flexural strength by $150 \%$. Deflection of reinforcement geopolymer composite increased by $180 \%$ against pure geopolymer mixture for both fiber types. Fig. 3 shows internal structure of geopolymer composite with glass fibre reinforcement.

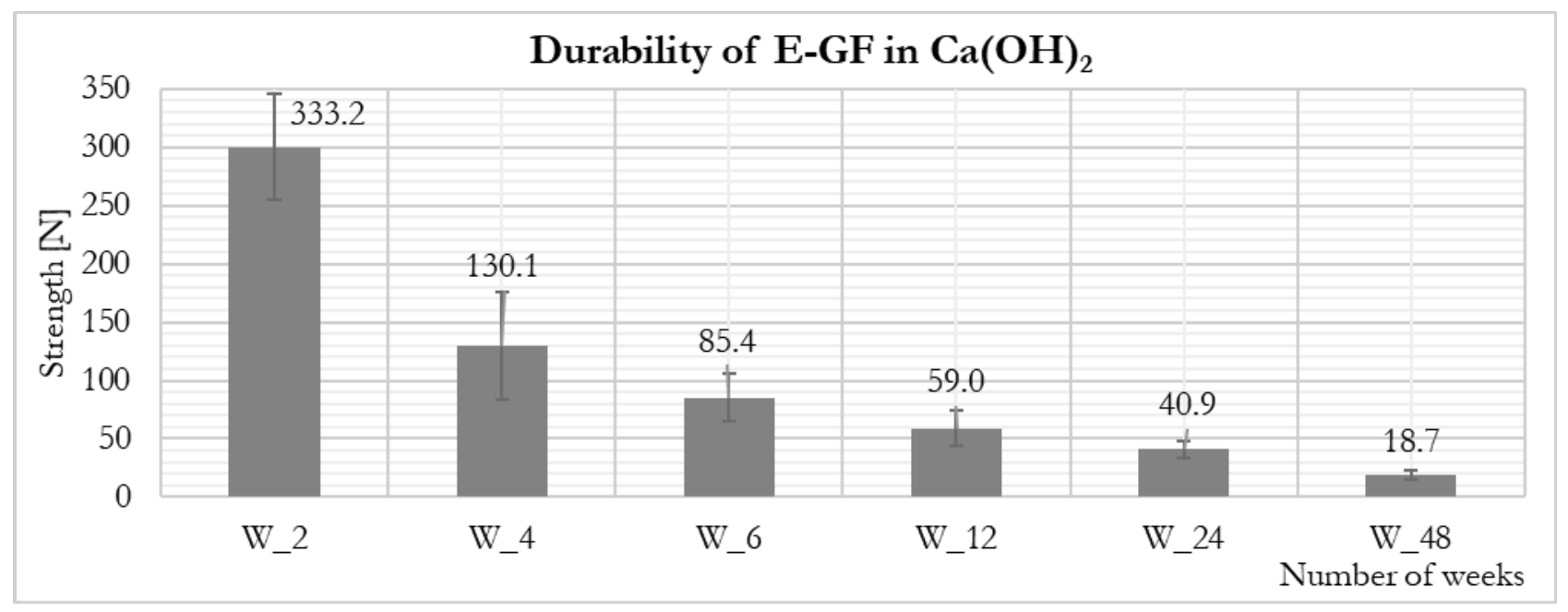

Fig. 2 Representation of the trend of degradation processes for $\mathrm{E}-\mathrm{GF}$ s in alkaline solution $\mathrm{Ca}(\mathrm{OH})_{2}$ in determined weeks

Tab. 2 Results of 3point bending test

\begin{tabular}{|l|l|l|l|}
\hline Samples & Fmax [N] & Deflection $[\mathrm{mm}]$ & $\sigma[\mathrm{MPa}]$ \\
\hline pure & $22.0 \pm 4.7$ & $0.6 \pm 0.1$ & $3.44 \pm 1.23$ \\
\hline with E-GF & $50.8 \pm 18.8$ & $1.1 \pm 0.2$ & $4.18 \pm 2.74$ \\
\hline with AR-GF & $44.9 \pm 8.3$ & $1.2 \pm 0.4$ & $4.90 \pm 0.96$ \\
\hline
\end{tabular}



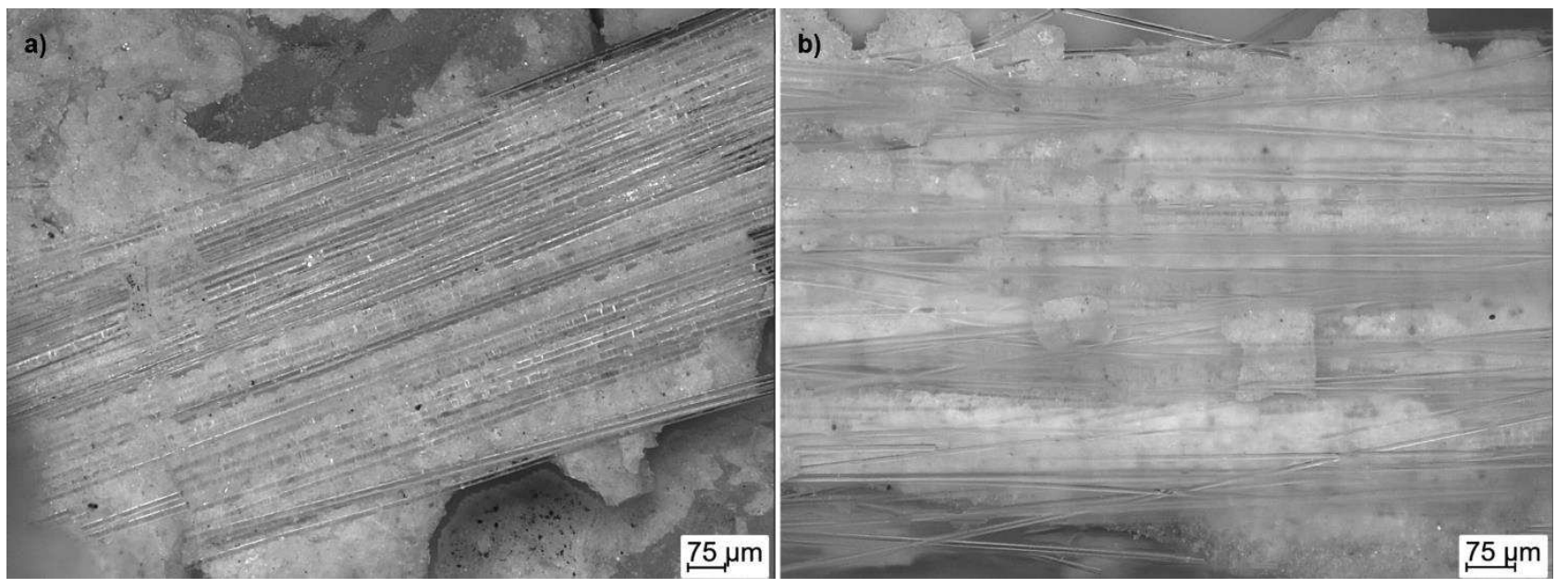

Fig. 3 Internal structure of geopolymer composite with a) AR-GFs reinforcement, b) E-GFs reinforcement

\subsection{Image analysis}

Generally, SEM analysis helps to determine changes on fibres surface after treatment in acid, alkaline and salt environment and investigation typical differences for different environment. Action of acid generate radial cracks after short time influence and axial cracks after influence long time [4, 5]. SEM analysis is important for observaion and description grow of corrosion shell for basalt and glass fibres; small differences exist between degradation both types of fibres [9].

For monitoring changes on fibre surface was used Scanning Electron Microscopy UHR FE-SEM Carl Zeiss ULTRA Plus. Figure 4a shows surface of ARGFs after 6 weeks in $1 \mathrm{~mol}$ liquid $\mathrm{Ca}(\mathrm{OH})_{2}$ with coating (sizing), which protect fibre surface against aggres- sive influence of alkaline liquid or environment. Similar result is de-cribed in study [7]. Authors observe influence of interphase modification of AR-glass fibres durability in alkaline environment. Degradation process is very intensive, corrosion shell has created during the exposure time and sizing has removed. Degree of degradation was determined according results of tensile strength test of elementary glass fibrils, for evaluation was used Weibull distribution function. Study [2] confirms protective function of sizing depend on sizing amount. For investigation was used tensile strengthtest, SEM analysis and AFM method for topology of fibre surface. In the study [10], changes in the AR-GF surface were monitored by SEM analysis. Strong alkaline environment (represented $\mathrm{NaOH}$ and $\mathrm{Ca}(\mathrm{OH})_{2}$ solutions) disturbs surface of AR glass fibres, holes create after long term exposition.

Tab. 3 Results of EDX analysis

\begin{tabular}{|l|l|l|l|l|l|l|l|l|}
\hline Spectrum label & $\mathrm{O}$ & $\mathrm{Si}$ & $\mathrm{Al}$ & $\mathrm{Na}$ & $\mathrm{B}$ & $\mathrm{Zr}$ & $\mathrm{Ca}$ & $\mathrm{Fe}$ \\
\hline AR-GF & 63.7 & 21.0 & - & 10.4 & - & 2.8 & 2.1 & - \\
\hline E-GF & 54.2 & 20.5 & 12.4 & - & 2.1 & - & 12.1 & 0.2 \\
\hline
\end{tabular}
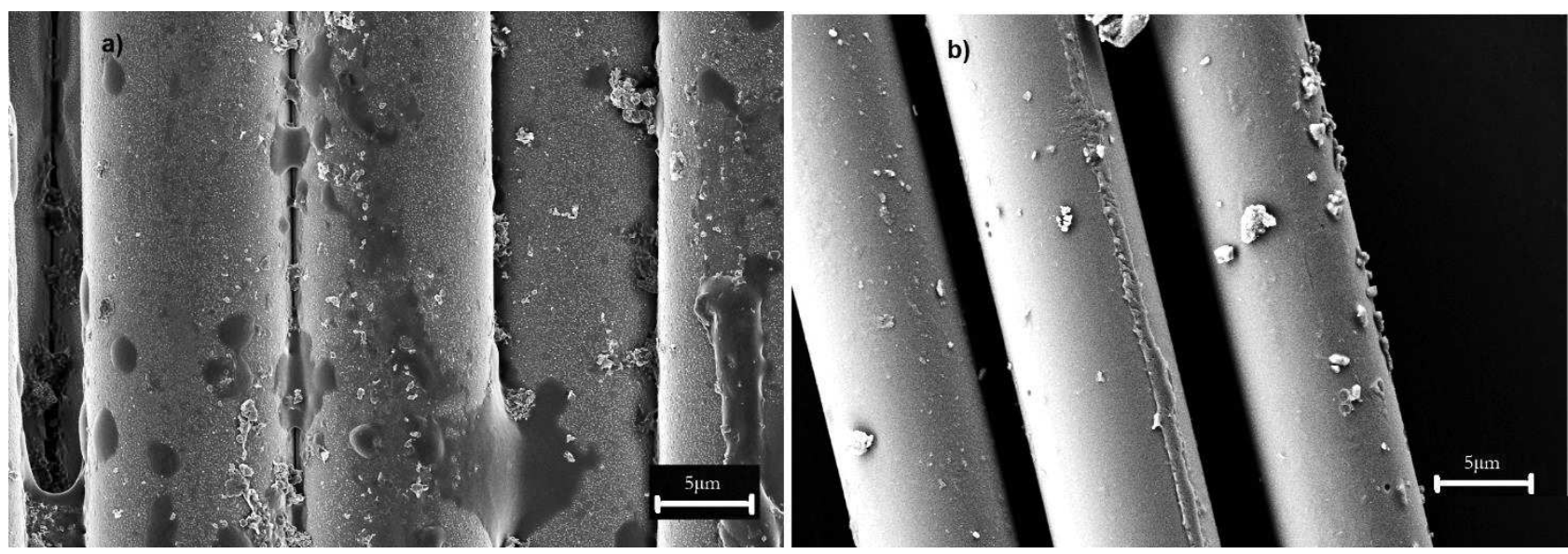

Fig. 4 Illustration/Representation of fiber surfaces using SEM a) AR-GFs, b) E-GFs 

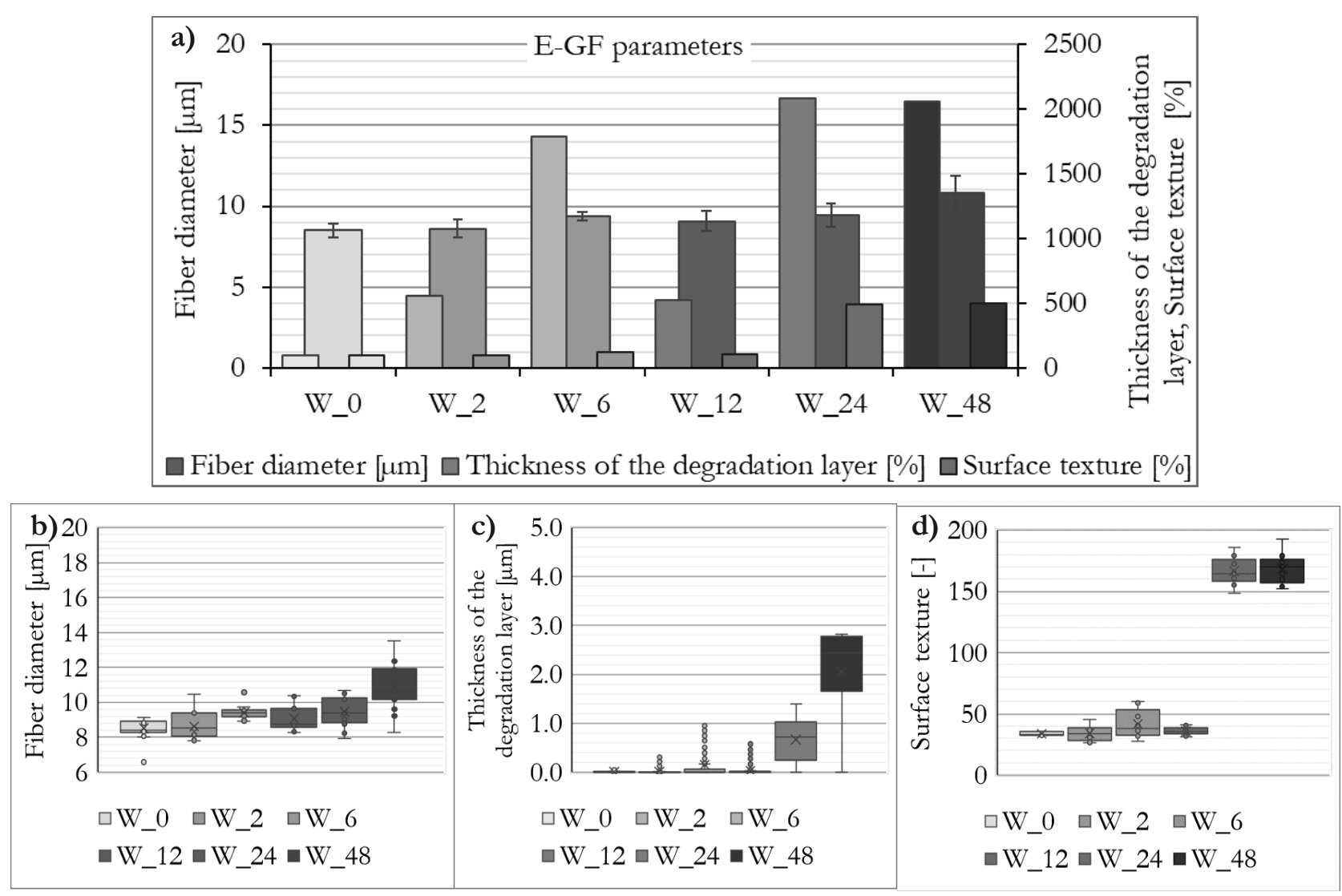

Fig. 5 (a) Image analysis of glass fibre type of E-GF, documented average changing of fibre properties within 48 weeks washed in $\mathrm{Ca}(\mathrm{OH})_{2}$ solution. Box plot show average values (as lines inside of boxes), variance values (as the top or bottom edge of the box) and also the outlier's data (as isolated dots) for (b) the fibre diameter, (c) thickness of the degradation layer, (d) surface texture

Figure 4b shows smooth surface of E-GFs after 6 weeks in $1 \mathrm{~mol}$ liquid $\mathrm{Ca}(\mathrm{OH})_{2}$, there are small amount of sizing residues in the picture. Long-term influence of calcium-hydroxy solution at ambient temperature leads to the creation of corrosion shell on surface of E-glass fibres. SEM analysis of this process was described in study [3]. Grad-ually formation of corrosion coating decreased tensile strength of fibres significantly; after six months by $87 \%$, after twelve months by $94 \%$.

Durability of AR-GFs is based on their composition too, especially on the content of $\mathrm{ZrO}_{2}$. Composition of glass fibres present Table 3 , presence of elements and their weight ratio was determined and confirmed using the EDX-analysis.

Average values of parameters obtained by image analysis are show on Figure $5 \mathrm{a}$ and 6a. Measurement deviations are not shown (only for the evaluation of the fibre diameter are shown) due to significant inconsistencies occurring in the images (unwashed parts of the sizing, etc.). Box plot prepared for each parameter show average values (as lines inside of boxes), variance values (as the top or bottom edge of the box) and also the outlier's data (as isolated dots); please see Figure 5b, c, d, and Figure 6b, c, d.
The E-GFs type was tested within 48 weeks (W_0 correspond to beginning of experiment, W_48 correspond to 48 weeks and similar). Fibre diameter increase over time (gradually 8.5, 8.6, 9.4, 9.1, 9.5, 10.8 $\mu \mathrm{m})$. Average values of thickness of the degradation layer is greatly affected by outliers, thus more represented overview of process can by shown on Figure 5b. Extremely changes were identified in the week of 24 and 48, where the thickness of the layer reached increasing of about 0.8 and $2.2 \mu \mathrm{m}$ compared to sample W_0. Surface texture has similar progress as thickness determination. Only last two observations (24 and 48 weeks, Figure 5c) reach extreme values (more than four times higher than first four observations).

The AR-GFs type was tested within 6 weeks (W_0 correspond to beginning of experiment, W_6 correspond to 6 weeks washed in $\mathrm{Ca}(\mathrm{OH})_{2}$ solution). Fibre diameter changing over time (first grow slightly, then it decreases). Average values of thickness of the degradation layer is greatly affected by outliers, thus more represented overview of process can by shown on Figure $6 \mathrm{~b}$. The thickness of the degradation layer decrease within 6 weeks in $\mathrm{Ca}(\mathrm{OH})_{2}$ solution. Surface texture has special progress, where the values in time of 4 and 6 weeks are higher than values in the beginning (W_0). 


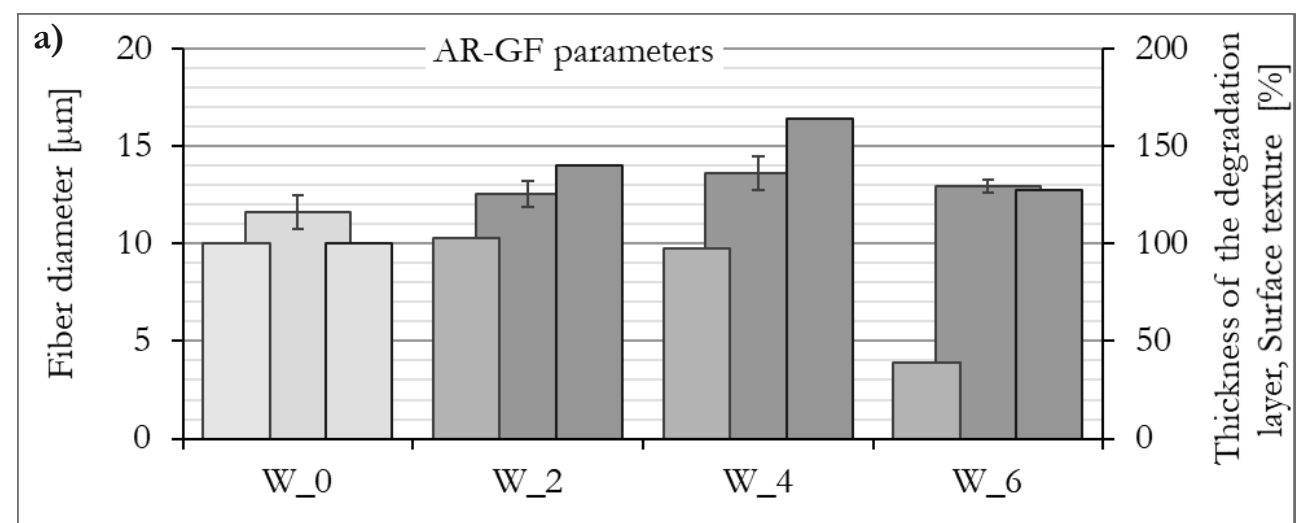

Fiber diameter $[\mu \mathrm{m}] \square$ Thickness of the degradation layer $[\%] \square$ Surface texture $[\%]$
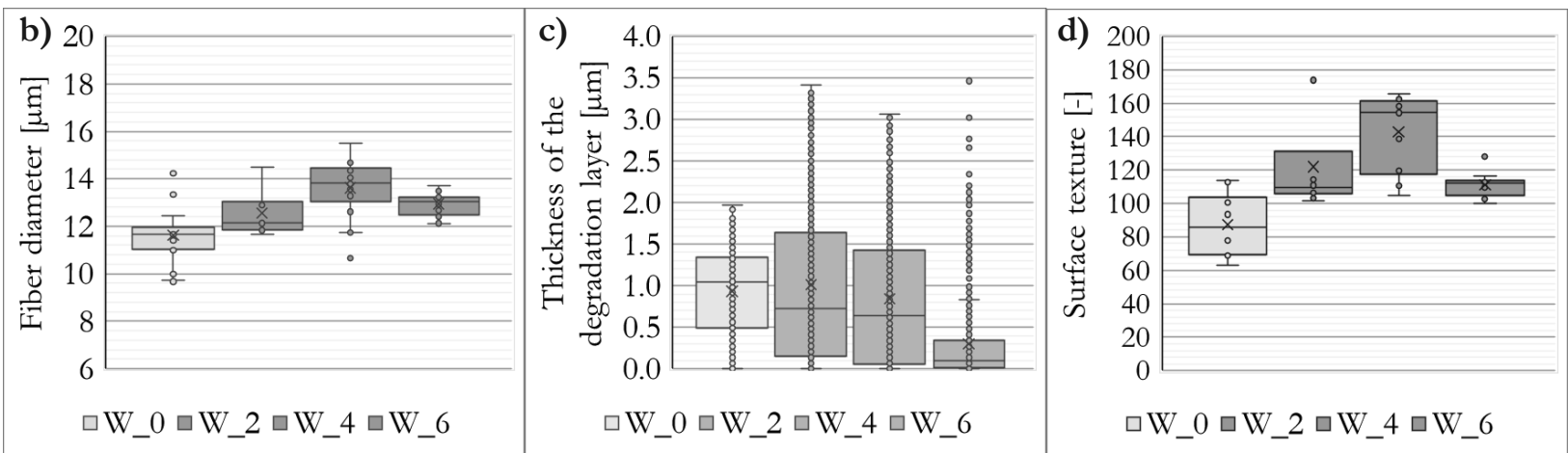

Fig. 6 (a) Image analysis of anticorrosive glass fibre documented average changing of fibre properties within 6 weeks. Box plot show average values (as lines inside of boxes), variance values (as the top or bottom edge of the box) and also the outlier's data (as isolated dots) for (b) the fibre diameter, (c) thickness of the degradation layer, (d) surface texture

\section{Conclusion}

The aim of the research is to compare the rates of degradation layer formation on the surface of AR (alkali-resistant glass composed of zirconium alkali silicates is used in cement substrates and concrete) and $\mathrm{E}$ (alumi-num-calcium-borosilicate glasses used as multipurpose fibres) fibres due to alkaline environment both on separate fibres, as well as in the volume of geopolymer composites.

Due to $1 \mathrm{~mol} \mathrm{Ca}(\mathrm{OH})_{2}$ liquids on E-GFs, it led to a rapid decrease in the tensile strength of the fibers. Af-ter two weeks, the mechanical properties of E-GFs decreased by $64 \%$. Long-term influence of alkaline environmet caused next decrease strength of E-GFs. Strength of roving decreased against reference strength after 12 weeks by $93 \%$, after 48 weeks by $98 \%$. Strength of roving between samples after 2 weeks and after 12 weeks decreased by $80 \%$. Diference between strength of roving at 2 weeks and strength of roving after 48 weeks was $94 \%$.

The use of glass fibers as a reinforcement in composites has been shown to increase its flexural strength by $56 \%$ for AG-GFs and by $50 \%$ for E-GFs.

Further, our paper confirms a correlation between mechanical properties and surface observation (based on SEM microscopy and image analysis). When the mechanical strength declines (E-GFs), the fiber diameter in-crease, surface texture has more prominent texture and the thickness of degradation layer increase at the same time as well (linear relationship with mechanical strength).

In the future, this research will focus on monitoring the rate of degradation of various types of fibers with the help of image analysis under the action of ultraviolet and electromagnetic radiation.

\section{Acknowledgement}

This work was supported by the Student Grant Competition of the Technical University of Liberec under the project No. SGS-2020-5019.

The work was supported by the Ministry of Industry and Trade in the framework of the targeted support of the Application, Call VII, the Operational Program Enterprise and Innovations for Competitiveness - "Research and development of textile products focusing on knitting technology and using aqueous chemicals", reg. number CZ.01.1.02/0.0/0.0/19_262/0020121.

\section{References}

[1] HARTMAN, D., GREENWOOD, M.E., MILLER, D.M. High Strength Glass Fibres, 
Technical papers AGY, Pub.No.LIT-2006-111, USA

[2] MADER, E., PLONKA, R., SCHIEKEL, M., HEMPEL, R. (2004) Coatings on Alkali-resistant Glass Fibres for the Improvement of Concrete, Journal of Industrial Textiles, Vol. 33, No. 3

[3] RYVOLOVÁ, M., ŠKODOVÁ, M., LOUDA, P., VOLESKÝ, L. (2020) Study of glass fibres degradation in alkaline environment using image analysis, Fine Mechanics and Optics, Vol. 4, ISSN 0447-6441

[4] QIU, Q., KUMOSA, M. (1997) Corrosion of E-Glass fibers in acid environments, Composites Science and Technology 57 497-507

[5] EHRENSTEIN, G. W. (2009) Polymers composite materials. Prague: Scientia, ISBN 9788086960296

[6] KOLÍNOVÁ, M.a kol. (2019) Non-destructive Evaluation of Pore Size and Structural Change in Geopolymer Composite Materials with Added Fibres, Manufacturing Technology, Vol. 19, No. 1, ISSN 1213-2489

[7] SCHEFFLER, C, FÖRSTER, T, MÄDER, E, et al (2009) Aging of alkali-resistant glass and basalt fibres in alkaline solutions: Evaluation of the failure stress by Weibull distribution function. Journal of Non-Crystalline Solids 355:25882595. https://doi.org/10.1016/j.jnoncrysol.2009.09.018

[8] TANG, C, JIANG, H, ZHANG, X, et al (2018) Corrosion Behavior and Mechanism of Basalt Fibres in Sodium Hydroxide Solution. Materials 11:1381.

https://doi.org/10.3390/ma11081381

[9] NASIR, V., DOOTEJTEMA, A., (2012) Comparison between the Corrosion Mechanism and Crack Formation of Basalt and Glass Fibers in Aggressive Media, Eccm15 - 15th European Conference on Composite Materials, Venice, Italy

[10] KOPECSKÓ, K. (2004). Durability of Glass Fibres, Conference: 6th International RILEM Symposium on Fibre-Reinforced Concretes (FRC), At Varenna, Italy, Volume: RILEM PRO39, 2004

[11] GAO, X, GILLESPIE, JW, JENSEN, RE, et al (2015) Effect of fibre surface texture on the mechanical properties of glass fibre reinforced epoxy composite. Composites Part A: Applied Science and Manufacturing 74:10-17. https://doi.org/10.1016/j.compositesa.2015.03.023

[12] PALMER, S, HALL, W (2012) Surface evaluation of carbon fibre composites using wavelet texture analysis. Composites Part B: Engineering 43:621-626. https://doi.org/10.1016/j.compositesb.2011.08.024

[13] COUSIN, P, HASSAN, M, VIJAY, P, et al (2019) Chemical resistance of carbon, basalt, and glass fibres used in FRP reinforcing bars. Journal of Composite Materials 53:3651-3670. https://doi.org/10.1177/0021998319844306

[14] WEI, B, CAO, H, SONG, S (2010) Tensile behavior contrast of basalt and glass fibres after chemical treatment. Materials \& Design 31:4244-4250.

https://doi.org/10.1016/j.matdes.2010.04.009

[15] RAMAN, R, GUO, F, AL-SAADI, S, et al (2018) Understanding Fibre-Matrix Degradation of FRP Composites for Advanced Civil Engineering Applications: An Overview. Corros Mater Degrad 1:27-41

[16] SZCZYPINSKI, M. a kol. (2018) Evaluation of Mechanical Properties of Composite Geopolymer Blocks Reinforced with Basalt Fibres, Manufacturing Technology, Vol. 18, No. 5, ISSN 12132489

[17] The MathWorks, Inc. (2019) Image Processing Toolbox. In: Image Processing Toolbox. https://www.mathworks.com/products/image.html. Accessed 13 Aug 2019

[18] ISO 11566:1996, Carbon fibre - Determination of the tensile properties of single-filament specimens, https://www.iso.org

[19] ISO 14125 Fibre-reinforced plastic composites - Determination of flexural properties, https://www.iso.org

[20] ČSN EN 14617- 2 Agglomerated stone - Slabs and tiles for wall finisches (internal and external), Determination of flexural properties, http:// csnonlinefirmy.agentura-cas.cz

[21] ČSN EN 14617-9 Agglomerated stone - Slabs and tiles for wall finisches (internal and external), Determination of impact resistance, http:// csnonlinefirmy.agentura-cas.cz

[22] EN 10045-1 (1990) Metallic materials - Charpy impact test - Part 1: Test method, http://csnonlinefirmy.agentura-cas.cz

[23] ČSN ISO 695 (ISO 3585:1998) Borosilicate glass $\quad 3,3 \quad-\quad$ Properties, http://csnonlinefirmy.agentura-cas.cz

[24] ASTM D2343-17 Standard Test Method for Tensile Properties of Glass Fiber Strands, Yarns, and Rovings Used in Reinforced Plastics, https://www.astm.org 